\title{
The pursuit of happiness
}

\author{
Researchers have struggled to identify how certain states of mind influence \\ physical health. One biologist thinks he has an answer.
}

\author{
BY JO MARCHANT
}

W hen Steve Cole was a postdoc, he had an unusual hobby: matching art buyers with artists that they might like. The task made looking at art, something he had always loved, even more enjoyable. "There was an extra layer of purpose. I loved the ability to help artists I thought were great to find an appreciative audience," he says.

At the time, it was nothing more than a quirky sideline. But his latest findings have caused Cole - now a professor at the Cousins Center for Psychoneuroimmunology at the University of California, Los Angeles - to wonder whether the exhilaration and sense of purpose that he felt during that period might have done more than help him to find homes for unloved pieces of art. It might have benefited his immune system too.

At one time, most self-respecting molecular biologists would have scoffed at the idea. Today, evidence from many studies suggests that mental states such as stress can influence health. Still, it has proved difficult to explain how this happens at the molecular level - how subjective moods connect with the vastly complex physiology of the nervous and immune systems. The field that searches for these explanations, known as psychoneuroimmunology (PNI), is often criticized as lacking rigour. Cole's stated aim is to fix that, and his tool of choice is genome-wide transcriptional analysis: looking at broad patterns of gene expression in cells. "My job is to be a hard-core tracker," he says. "How do these mental states get out into the rest of the body?"

With his colleagues, Cole has published a string of studies suggesting that negative mental states such as stress and loneliness guide immune responses by driving broad programs of gene expression, shaping our ability to fight disease. If he is right, the way people see the world could affect everything from their risk of chronic illnesses such as diabetes and heart disease to the progression of conditions such as HIV and cancer. Now Cole has switched tack, moving from negative moods into the even more murky territory of happiness. It is a risky strategy; his work has already been criticized as wishful thinking and moralizing. But the pay-off is nothing less than finding a healthier way to live.

"If you talk to any high-quality neurobiologist or immunologist about PNI, it will invariably generate a little snicker," says Stephen Smale, an immunologist at the University of California, Los Angeles, who is not affiliated with the Cousins Center. "But this doesn't mean the topic should be ignored forever. Someday we need to confront it and try to understand how the immune system and nervous system interact."

\section{THE BEST MEDICINE?}

In 1964, magazine editor Norman Cousins was diagnosed with ankylosing spondylitis, a lifethreatening autoimmune disease, and given a 1 in 500 chance of recovery. Cousins rejected his doctors' prognosis and embarked on his own programme of happiness therapy, including regular doses of Marx Brothers films, and credited it with triggering a dramatic recovery. He later established the Cousins Center, which is dedicated to investigating whether psychological factors really can keep people healthy.

At the time, mainstream science rejected the idea that any psychological state, positive or negative, could affect physical well-being. But studies during the 1980s and early 1990s revealed that the brain is directly wired to the immune system - portions of the nervous system connect with immune-related organs such as the thymus and bone marrow, and immune cells have receptors for neurotransmitters, suggesting that there is crosstalk.

\section{"Mood matters. If we change the psychology, physiological changes do parallel that."}

These connections seem to have clinical relevance, at least in the case of stress. One of the first researchers to show this was virologist Ronald Glaser, now director of the Institute for Behavioral Medicine Research at the Ohio State University in Columbus. "When I started working on this in the 1980s, nobody believed what stress could do, including me," he recalls. He and his colleagues sampled blood from medical students, and found that during a stressful exam period, they had lower activity from virus-fighting immune cells ${ }^{1}$, and higher levels of antibodies for the common virus Epstein-Barr ${ }^{2}$, suggesting that stress had compromised their immune systems and allowed the normally latent virus to become reactivated.

The field of PNI has grown hugely since then, with medical schools worldwide boasting their own departments of mind-body medicine, of which PNI is just one component. It is now accepted that the body's response to stress can suppress parts of the immune system and, over the long term, lead to damaging levels of inflammation. Large epidemiological studies - including the Whitehall studies, which have been following thousands of British civil servants since 1967 - suggest ${ }^{3}$ that chronic work stress increases the risk of coronary heart disease and type 2 diabetes, for example. Low socio-economic status increases susceptibility to a wide range of infectious diseases, and there is considerable evidence that stress increases the rate of progression of HIV/AIDS. But researchers have a long way to go before they will understand exactly how signals from the brain feed into physical health.

\section{WORRIED SICK}

PNI studies have mostly tended to look at levels of individual immune-cell types or molecular messengers - such as the stress hormone cortisol and the immune messenger proteins called cytokines - or the expression of individual genes. But Cole wanted to get a sense of how the whole system was working.

His first foray, published in 2007 , looked at loneliness ${ }^{4}$. Social isolation is one of the most powerful known psychological risk factors for poor health, but it is never certain whether it causes the health problems, or whether a third factor is involved: lonely people might be less likely than others to eat well, for example, or to visit their doctor regularly.

Cole and his colleagues looked at gene expression in the white blood cells of six chronically lonely people - people who had said consistently over several years that they felt lonely or isolated, and were fearful of other people - and eight people who said that they had great friends and social support. Out of the roughly 22,000 genes in the human genome, the researchers identified 209 that distinguished the lonely people from the sociable ones: they were either regulated up to produce 


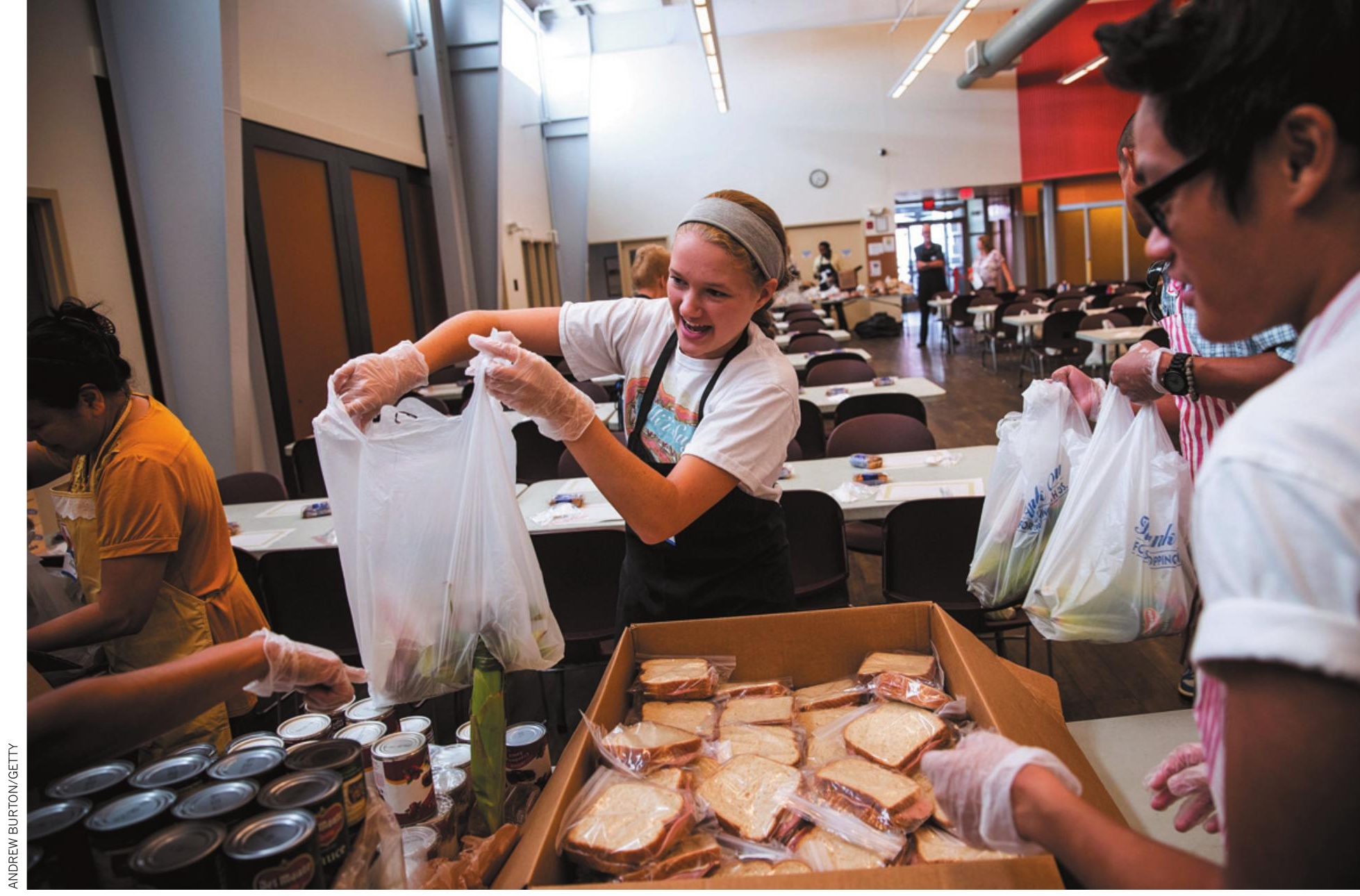

A volunteer helps to bag meals for the homeless at Cathedral Kitchen in Camden, New Jersey.

more of an individual protein or regulated down to produce less. Any individual gene could easily look different by chance, but Cole was struck by the overall pattern. A particularly large proportion of the upregulated genes in the lonely group turned out to be involved in the inflammatory response, whereas many of the downregulated genes had antiviral roles. In sociable people, the reverse was true. It was a small study, but one of the first to link a psychological risk factor with a broad underlying change in gene expression.

The researchers have since replicated that result in a group of 93 people ${ }^{5}$. Cole says that he has also seen a similar shift in gene expression in individuals exposed to various types of social adversity, from imminent bereavement to low socio-economic status.

The results make evolutionary sense, he says. Early humans in close-knit social groups would have faced increased risk of viral infections, so they would have benefited from revved-up antiviral genes. By contrast, people who were isolated and under stress faced greater risk of injuries that could cause bacterial infection - and thus would need to respond by ramping up genes associated with inflammation, to help heal wounds and fight off those infections. But modern stresses lead to chronic and unhelpful inflammation, which over time damages the body's tissues, increasing the risk of chronic diseases such as atherosclerosis, cancer and diabetes.

To a classical immunologist such as Smale,
Cole's results are "intriguing, wonderful observations", but not yet completely convincing. In future work, he wants to see the rest of the physiological pathway nailed down. "Until you put together a full understanding of that mechanism, you have this level of uncertainty and scepticism," he says. That sentiment is echoed by Alexander Tarakhovsky, an immunologist at the Rockefeller University in New York City. Pinning down precise mechanisms - for example, which neurotransmitters cause which specific effects - is extremely difficult, he says, because the brain and the immune system are both so complex. Cole's research "makes you think about what the consequences of social hardship could be, but it doesn't really tell you how it works".

Greg Gibson, director of the Center for Integrative Genomics at the Georgia Institute of Technology in Atlanta, wants to see larger studies but argues that the big-picture "genetic architecture" that Cole is uncovering is worth studying, even if not every detail of the mechanism is yet understood. "A lot of people are taking a whole-genome approach, but they focus only on a handful of 'top hits.' They are missing the wood for the trees."

\section{DON'T WORRY, BE HAPPY}

In 2010, Cole received an e-mail from Barbara Fredrickson, a friend from graduate school who was now studying emotional wellbeing at the University of North Carolina in Chapel Hill. "Remember me?" she said. She was interested in the biological correlates of happiness and other positive emotional states, and suggested that the pair collaborate. After years of looking at stress and adversity, Cole loved the idea. "I was bored as hell with misery," he says.

If PNI as a whole has credibility issues, studying well-being is even trickier. It is more slippery to measure than stress - there is no biological marker such as cortisol to fall back on and no simple way to induce it in the lab, and mainstream biologists tend to look down on fuzzy methods of data collection such as questionnaires.

One approach is to test whether it is possible to reverse the adverse effects on gene expression caused by stress. Cole has collaborated in three small, randomized, controlled trials that attempt to do this. Studies involving 45 stressed caregivers $^{6}$ and 40 lonely adults ${ }^{7}$ respectively found that courses in meditation shifted gene-expression profiles in the participants' white blood cells away from inflammatory genes and towards antiviral genes. A third trial ${ }^{8}$, led by psycho-oncologist Michael Antoni at the University of Miami, Florida, involved 200 women with early-stage breast cancer. In those who completed a tenweek stress-management programme, genes associated with inflammation and metastasis were downregulated compared with those of women in the control group, who attended a one-day educational seminar. Meanwhile, genes involved in the type I interferon response 
